\title{
Chapter 14 \\ Hyperspectral Stochastic Optical \\ Reconstruction Raman Microscopy for \\ Label-Free Super-Resolution Imaging Using \\ Surface Enhanced Raman Spectroscopy
}

\author{
Aykutlu Dâna
}

\begin{abstract}
Super-resolution imaging is an emerging field that has attracted attention in the recent years due to far the reaching impact in biology. All super-resolution techniques use fluorescent labels to image nanoscale biomolecular structures. In contrast, label-free nanoscopic imaging of the chemical environment of biological specimens would readily bridge the supramolecular and the cellular scales, if a chemical fingerprint technique such as Raman scattering can be coupled with superresolution imaging, overcoming the diffraction limit. In order to achieve this goal, we propose to develop a super-resolved stochastic hyperspectral Raman microscopy technique for imaging of biological architectures. The surface enhanced Raman spectroscopy (SERS) signal contains information about the presence of various Raman bands, allowing for the discrimination of families of biomolecules such as lipids, proteins, DNA. The rich, fluctuating spectral information contained in the single molecule SERS signal possesses a great potential in label-free imaging, using stochastic optical reconstruction microscopy (STORM) methods. In a recently published work, we demonstrated $20 \mathrm{~nm}$ spatial resolution using the spectrally integrated Raman signal on highly uniform SERS substrates. A mature version of our method would require development of spectrally resolved nanoscale Raman imaging. Development of stochastic Raman imaging addresses the issue by design and construction of a Raman microscope with hyperspectral imaging capability that will allow imaging of different Raman bands of the SERS signal. Novel computational techniques must also be developed that will enable extraction of hyperspectral STORM images corresponding to different Raman bands, while simultaneously allowing conventional STORM data to be collected using the wellestablished labelling techniques. The resulting technique (Hyperspectral Raman
\end{abstract}

\footnotetext{
A. Dâna $(\bowtie)$

UNAM Institute of Materials Science and Nanotechnology, Bilkent University, 06800 Ankara, Turkey

e-mail: aykutlu@unam.bilkent.edu.tr
} 
STORM or HyperSTORRM) has the potential to complement the available labeled stochastic imaging methods and enable chemically resolved nanoscopy.

\subsection{Introduction}

In this chapter, we discuss issues relating to the development of an advanced imaging technique that would allow nanometer scale resolution far-field imaging of biological architectures with the chemical specificity present in the Raman scattered optical signal. Specifically, the method uses stochastic reconstruction methods on the fluctuating optical signal present in single-molecule surface-enhanced Raman phenomena to achieve this goal.

Raman microscopy can be applied in a confocal configuration, to provide Raman maps of surfaces. Raman microscopy using pulsed illumination resulted in CARS (coherent anti-stokes Raman) and SRS (stimulated Raman) microscopy, also typically exhibiting diffraction limited resolution [1]. Similarly, confocal or wide-field Raman imaging displays a resolution of $\sim 250 \mathrm{~nm}$ at best. Nearfield microscopy using tip-enhanced Raman microscopy provides nanometer scale resolution, however, the technique is slow and is not far-field, because it requires nanometer separation between a scanning tip and the sample, and can't be applied for 3D imaging [2]. Plasmonic structures made the acquisition of Raman spectra possible from sub-monolayer coverage molecular films, a technique known as Surface-Enhanced Raman Spectroscopy (SERS) [3-5]. Using SERS, single molecule level sensitivity can be achieved [6-9]. This was previously attributed to extremely high enhancement factors of $10^{9}-10^{12}$. It was later claimed that much lower enhancement factors $\left(10^{6}-10^{8}\right)$ may be sufficient for such observations [10]. Stochastic optical reconstruction microscopy (STORM) is a versatile technique that allows sub-diffraction limited resolution through mathematical analysis of a video sequence of blinking fluorescence signals in a sample. The centroids of blinking spots are calculated using various algorithms to achieve the reconstruction. Traditionally, photoactivated or self-blinking fluorescent molecular labels are used in STORM to highlight various cellular architectures. Labeling allows discrimination of components, however potentially disturbs the native state of the biomolecular structures. The SERS signal, at single-molecule level, typically shows a rapid blinking behavior which can be captured with a camera or spectrometer. This nonstationary nature of the SERS signal and the rich spectral information contained in the acquired spectra, in principle, provides an opportunity to exploit the SERS signal for chemically-resolved, label-free, super-resolution stochastic imaging [11]. Widefield imaging and stochastic reconstruction methods have been applied to observe the spatial distribution of the of the SERS signal, allowing reconstruction of the field distributions of individual hot-spots [12]. Traditionally, similar reconstruction methods have used fluorescence signal from single molecules and nanoparticles, relying on photo-activation, photo-bleaching, and molecular diffusion [13]. An increasing number of articles used wide-field imaging and stochastic analysis of 
the SERS signal as an application of SERS in super-resolved imaging of field distributions hot-spots [14]. However, for SERS to be a viable way of reconstructing the nanoscale structure of a biomolecular layer, rather than the field distribution of the underlying hot-spot itself, a large number of closely packed hot-spots is required within a focal spot with an area defined by the diffraction limit. In this work, we take a completely novel approach to Raman imaging. Previously, meta-surfaces with periodicities larger than the diffraction limited spot size have been shown to produce high and uniform enhancement [15]. Recently, we used an engineered meta-surface with sub-wavelength periodicity to demonstrate high uniformity and high spatial resolution confocal SERS imaging [16]. In following work, the plasmonic designs are extended and techniques for production of easy-tofabricate SERS substrates are developed that exhibit a high density of hot-spots while allowing single-molecule level Raman spectroscopy [17, 18]. SERS and STORM techniques are combined to produce reconstructed images of biomolecular structures. The enhancement uniformity and performance featured by the substrate is sufficient to perform STORM with $20 \mathrm{~nm}$ resolution [17]. In this chapter, hyperspectral stochastic imaging using the SERS signal and the development of a label free nanoscopic imaging technique is discussed.

\subsection{Basic Description of the Hyperspectral Super-Resolved Raman Imaging System}

Figure 14.1 shows the main idea of a Hyperspectral Stochastic Optical Reconstruction Raman Microscopy (HYPERSTORRM). A hyperspectral wide-field Raman imaging system is described that can record SERS blink events with distinct spectral characteristics, belonging to different vibrational bands of biomolecules. Advanced stochastic reconstruction algorithms are needed to obtain nanometer resolution images that show chemical composition of biological architectures with nanometer resolution. In this imaging mode, the samples are placed on highlyuniform SERS substrates that exhibit single-molecule level sensitivity. In the basic version of the HYPERSTORRM microscope, a fixed excitation wavelength is used, along with multiple cameras (multispectral imaging module). Four Raman bands corresponding to common Raman active modes of biological structures was be selected during an experiment (by proper choice of dichroic mirrors and narrowband filter wavelengths) and initial images contained chemical information based on four Raman frequencies. In the second variation of the microscope, a hyperspectral imaging module is used (optical scheme shown in Fig. 14.2). The advantage of using a hyperspectral module is recording of the complete SERS spectrum, enabling stochastic reconstructed imaging using all available Raman information. Pulsed (pico-nanosecond pulse width) source can be used to reduce sample damage.

In hyperspectral image acquisition using a 2D detector array, typically a $1 \mathrm{D}$ grating with a linear slit, is used to separate the information into one dimensional 

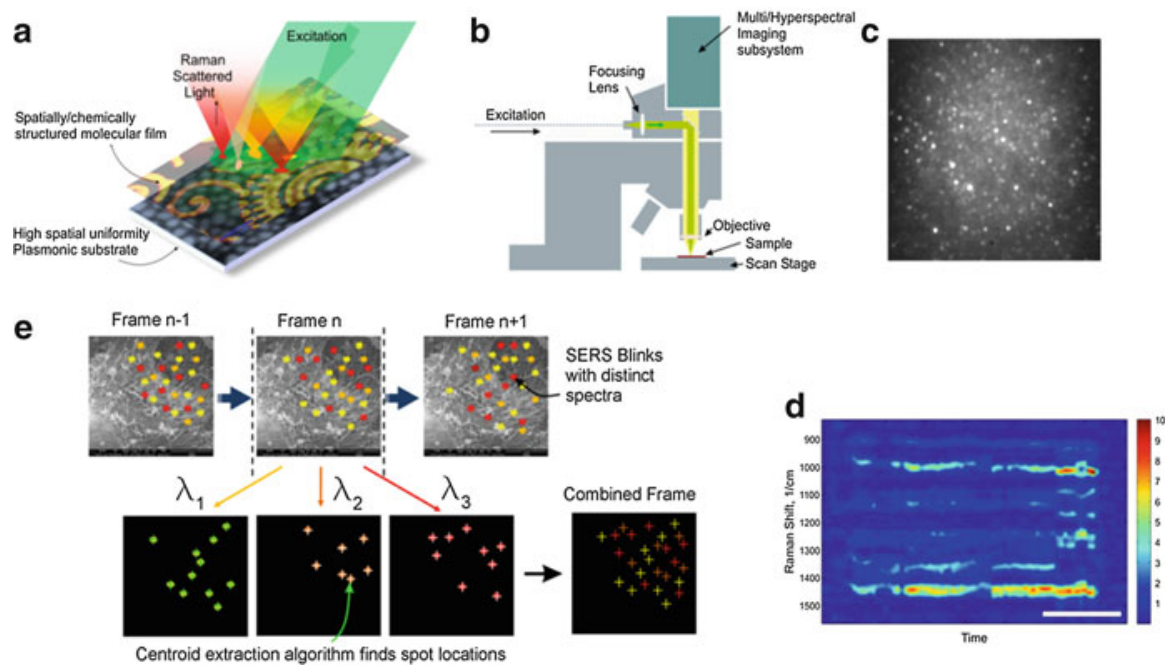

Fig. 14.1 Description of the HYPERSTORRM Microscope. (a) A biological sample (such as a cell membrane, self-assembled supramolecular structures, extra or intracellular matrix components) is placed on a high performance and high uniformity plasmonic substrate. Under wide-field excitation, SERS signal originates from the sample. (b) A Raman microscope system equipped with a multi/hyperspectral imaging module allows collection of light from the sample. (c) Example wide-field SERS image from a uniform plasmonic substrate covered with a uniform peptide layer is given, showing evenly distributed blinking spots. (Supporting information of [17] has videos showing SERS induced blinking in various samples). (d) Spectrum collected from a single blinking spot using a fiber shows typical characteristic fluctuations in the intensity and frequency of Raman bands during single-molecule blinking events. (e) HYPERSTORRM uses such fluctuations and their correlations to reconstruct a Raman map with nanometer scale resolution

spatial and spectral directions [19]. As the sample is scanned in one spatial direction, a hyperspectral map can be acquired. This conventional method of hyperspectral imaging is not adequate for stochastic super-resolution imaging. Instead, a way of reducing the three dimensional (two spatial and one spectral) data to the two dimensional space of the detector must be developed, without resorting to sample scanning. One has to either use a large number of spectrally separated cameras, or approximate the solution by imposing limitations on the optical signal. Indeed, it is shown in Sect. 4 that, by limiting the spatial extent of the image and spectral extent of the signal and by introducing the correlations present in fluctuations of the SERS signal, the problem of hyperstactral reconstruction can be solved. In order to clarify our approach, consider the scheme presented in Fig. 14.2, where the image is recorded using a 2D transmission grating in front of the image sensor, and 0th and higher order images are acquired. The center (0th order) image is used for localization. Using the localization data and by using time correlations of spectral features with the 0th order image pixels, one can extract the spectral content of the SERS signals and assign them to spots in the 0th order image. In this way, the hyperspectral super-resolution maps can be produced from SERS data. 

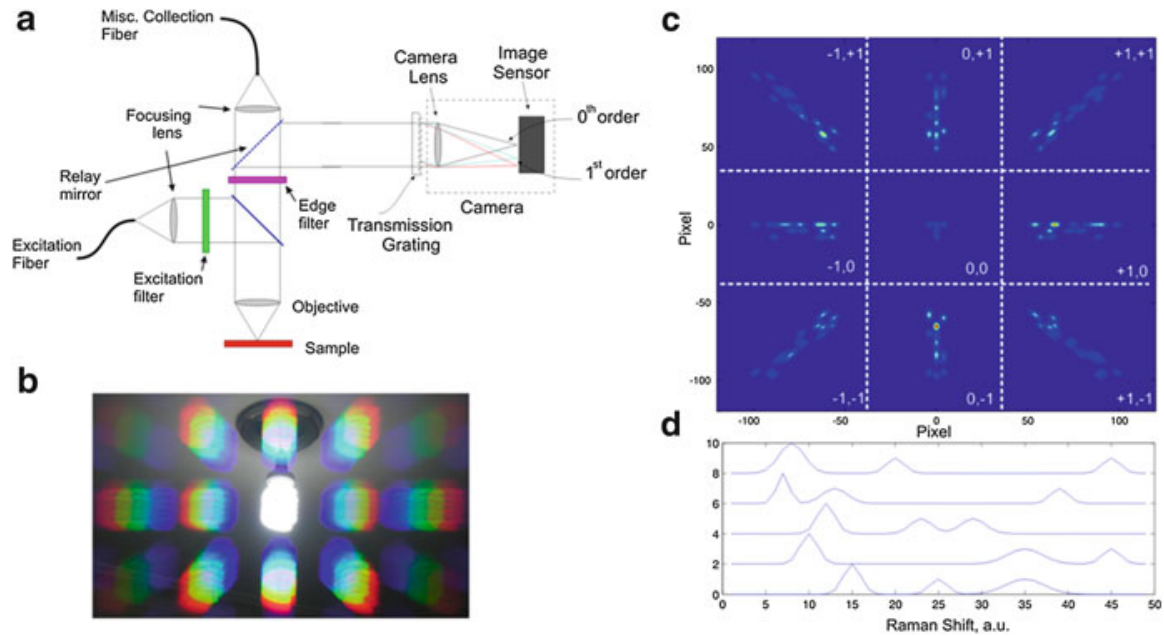

Fig. 14.2 Preliminary optical design for HYPERSTORRM imaging. (a) A Raman microscope is modified to incorporate a high sensitivity camera and high efficiency transmission grating to form a hyperspectral imaging system. (b) The 0th order of the grating produces a real image and higher orders produce spectrally resolved images. Example image of a fluorescent light bulb taken with a 2D grating in front of a camera is shown. (c) Simulation of imaging of a spectrally rich sample is shown. Five spots in the form of a ' $T$ ' (center of image) are assumed to have different spectra. The 2D grating separates the spectra in positive and negative orders. By limiting the field of view to a diameter of about $10 \mu \mathrm{m}$ and spectra to visible range, 0th and 1st order images are well separated. The 0th order image can be used for localization and spatial reconstruction. The 1st order images contain spectral information, which can be used by advanced mathematical methods for the extraction of spectral data. Mathematically it is not possible to fully invert the imaging scheme to reconstruct the spectral content. However, by introducing time as a variable, the problem can be inverted to give the spectra corresponding to each spot. (d) The hypothetical spectra of the scattering centers used in the simulation in (c) are shown. Such spectral separation necessitates the use of a high sensitivity camera, as the optical signal is spatially multiplexed in this scheme

The design can be built around a commercial microscope system, allowing confocal Raman microscopy. The microscope may be modified for excitation with multiple wavelengths, including continuous and pulsed laser sources. The multispectral imaging head can be designed to allow easy change of filter combinations for use with different excitation wavelengths. A careful initial study must be conducted for determination of common Raman bands that are present in samples of interest (cellular components, proteins, peptidic nanostructures, DNA, lipids etc.). The laser excitation sources must be chosen to minimize fluorescence effects, if possible. Once the Raman bands and excitation wavelengths are determined (important for first generation experiments), filter wavelengths are fixed. Initial phase of design must focus on optimization of the detection efficiency, while matching the microscope and Electron Multiplied (EM) CCD cameras, in terms of aperture and pixel size. Synchronization of the frames from multiple cameras at high frame and data capture rate needs to be achieved by using high performance multicore desktop computers. 


\subsection{Feasibility of Highly-Uniform Single-Molecule Level SERS Substrates}

Previous work on the design and fabrication of high-uniformity and single-molecule level sensitivity SERS substrates, necessary for the realization of HYPERSTORRM, demonstrates the feasibility of the approach presented here. In initial experiments with quarter micron scale plasmonic structures, high-uniformity SERS spectroscopy and confocal imaging were demonstrated in a recent publication [16]. A further scaled down version of high-uniformity SERS substrates achieved single-molecule level sensitivity [17]. Therefore, the feasibility of fabricating high spatial uniformity and high sensitivity Raman substrates is well demonstrated. It is necessary to investigate ways to further improve hot-spot density and uniformity. An obvious direction is further scaling down the plasmonic surfaces to give a hot spot spacing of about $10 \mathrm{~nm}$. However, this scaling would require advances in fabrication, and keeping the enhancement factors above $10^{8}$ is not intuitive.

The improvement of spatial uniformity and smaller characteristic dimension SERS substrates, while increasing the field enhancement factor, plays a crucial role in achieving HyperSTORRM images with improved signal-to-noise ratio and resolution. Previously, Metal-Insulator-Metal (MIM) structures that exhibit spatially uniform SERS enhancement were studied. These were fabricated by using electron beam lithography as shown in Fig. 14.3. A detailed study of the relation between geometry and plasmonic enhancement properties has been conducted, using computational tools as well as analytical calculations based on circuit models of such structures (Fig. 14.3c). The models are observed to reproduce experimental results fairly well (Fig. 14.3d), and a high spatial uniformity can be achieved on large scales (>250 nm, Fig. 14.3e, f). Nanoscale uniformity is harder to achieve in the given geometry, and there is inherent non-uniformity within a unit-cell of the surface (Fig. 14.3g). However, as the characteristic size of the plasmonic structures shrink, it is experimentally observed that a more uniform enhancement is present. The uniformity issue is not straight forward and deserves more careful study.

In our model plasmonic system, the primary requirement for high SERS enhancement is the presence of plasmonic resonances at both the excitation and scattering wavelengths. Also, for high resolution imaging, the structures are required to be omnidirectional, allowing use of high numerical aperture objectives. A simplified analytical model of the periodic MIM structure allows rapid estimation of the effect of geometrical parameters on resonant properties (Fig. 14.3c). It can be seen that, scaling the MIM widths down to $10 \mathrm{~nm}$ while keeping the resonances at given laser wavelengths $(532-785 \mathrm{~nm}$ ) would require thin dielectric layers to the order of few nanometers. Our process uses atomic layer deposition to achieve high uniformity and excellent thickness control, therefore we have been able to shrink down to about $30 \mathrm{~nm}$ island size (inset of Fig. 14.3g). The improved surfaces resulted in the collection of preliminary images shown in Fig. 14.4. 
a
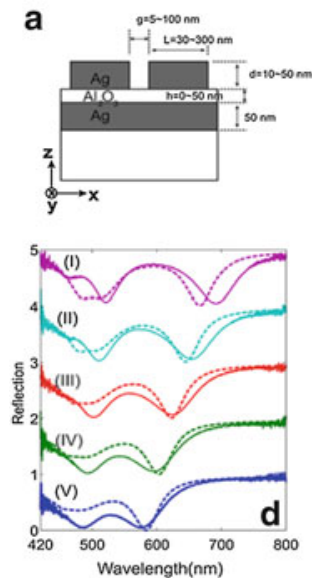

b
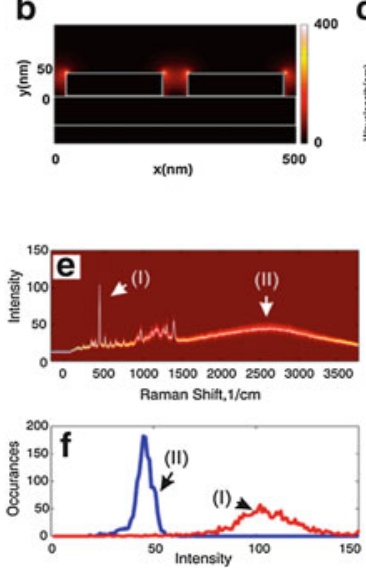
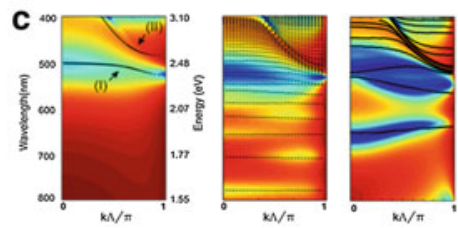

g

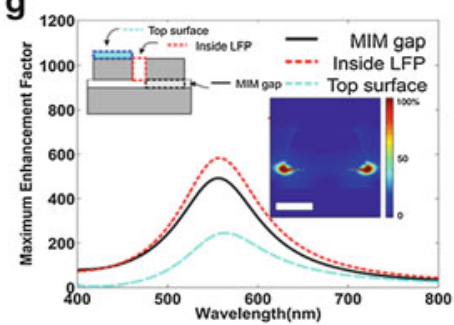

Fig. 14.3 Engineering high-performance plasmonic substrates. (a) Basic Metal-Insulator-Metal $(M I M)$ structure used for high uniformity SERS enhancement. (b) Typical enhancement profile (shown for a structure with diminishing dielectric thickness). (c) Band diagrams calculated for $0,3 \mathrm{~nm}$ and $10 \mathrm{~nm}$ dielectric thickness for $250 \mathrm{~nm}$ period structures. (d) Calculations and measurements of reflectance on surfaces prepared by electron beam lithography show good agreement. (e) 1600 SERS spectra collected over $10 \times 10 \mu \mathrm{m}$ area show excellent repeatability. (f) Histograms show 10-20\% uniformity of enhancement. (g) Shrinking the dimensions further down improves field enhancement and uniformity (inset shows fields for $40 \mathrm{~nm}$ period structures, scale bar $10 \mathrm{~nm}$ ). Enhancement factor shows a non-uniform distribution within the unit cell of the plasmonic surface, which prompts investigation of alternative structures or geometries

The surfaces are fabricated by spontaneous self-organization of Ag nanosilands during deposition of ultrathin Ag films on oxides. Due to the de-wetting of $\mathrm{Ag}$, the films do not conform the surface and form islands with diameters of about $20 \mathrm{~nm}$ and separations of about $10 \mathrm{~nm}$ (Fig. 14.5). When placed over an Ag layer coated with a dielectric such as $\mathrm{HfO} 2$, the surfaces become super-absorbers, spanning a large wavelength range which covers the Raman excitation and scattering wavelengths (Fig. 14.5b). When a SEM picture (inset of Fig. 14.5c) is used to derive a model surface, electromagnetic full field calculations can be used to estimate hot spot density and enhancement factor for a given wavelength (Fig. 14.5d). It is seen that our surfaces generate SERS enhancements with of $10^{9}$ around $700 \mathrm{~nm}$ (Fig. 14.5e) if the correct dielectric thickness is chosen. Hot-spot spacing of about $20 \mathrm{~nm}$ is achieved in such a configuration.

Note that, the image consists of signal from high enhancement regions (so called hot-spots) and improving hot-spot density results in a better sampling of the biomolecular structures. With sparse hot-spots, a continuous nanoscale map of the sample cannot be achieved. During the project we will investigate the effect of nanoscale non-uniformity on the imaging resolution while also looking for ways to reliably improve characteristic dimensions of the plasmonic structures towards $10 \mathrm{~nm}$ range. 
a

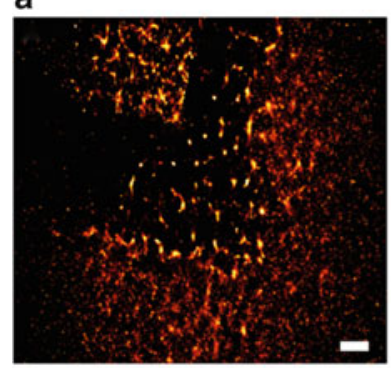

e b

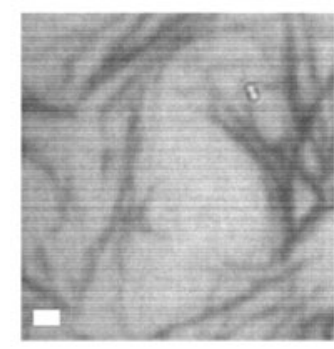

C

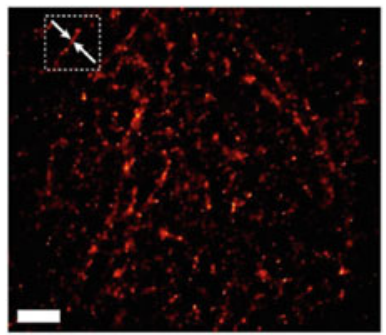

d

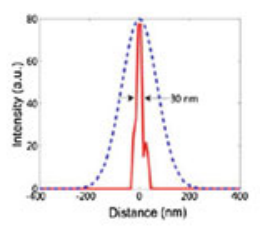

Fig. 14.4 Stochastic reconstruction SERS imaging of self-assembled peptide nanostructures: (a) Reconstruction of a peptide layer imprinted onto the plasmonic substrate using an elastomeric mold. (b) Bright field optical micrograph of a peptidic nanofiber network, directly self-assembled on the plasmonic substrate. (c) A wide-field SERS video sequence is recorded at the same location and resulting images are used to reconstruct image of self-assembled nanofiber network. Due to high SERS sensitivity, residual peptide presence on the surface complicates the resolution of individual peptide nanofibers. A well-separated, thin peptide nanobundle (highlighted by arrows) is used to plot the line profile. (d) The peptide bundle is observed as approximately $30 \mathrm{~nm}$ in diameter (solid line), a width smaller compared to the diffraction limited resolution (represented by dashed line). (e) During wide-field SERS video imaging of peptide nanofibers, SERS spectra was simultaneously recorded from a spot located at the center. Amide VI $\left(630-750 \mathrm{~cm}^{-1}\right)$, Amide V (700-750 $\left.\mathrm{cm}^{-1}\right)$, Amide III $\left(1,230-1,300 \mathrm{~cm}^{-1}\right)$, Amide II $\left(\sim 1,550 \mathrm{~cm}^{-1}\right)$ and Amide I $(1,600$ $1,700 \mathrm{~cm}^{-1}$ ) bands associated with peptide backbone structure and side chain composition can be identified in the spectra. ( $\delta$, in plane bending vibration; $v$, stretching vibrations.) (All scale bars are $1 \mu \mathrm{m}$ wide). Again, whole integrated Raman spectra are used with a low sensitivity CMOS camera for the reconstructions. Due to lack of spectrally resolved optics during the collection of this data set, the rich spectral information can't be fully used to reconstruct a chemically specific HYPERSTORRM image

\subsection{Feasibility of Stochastic Imaging Using the SERS Signal}

We have made initial demonstrations of HYPERSTORRM imaging using a low sensitivity CMOS camera, which uses the whole integrated Raman spectrum, on two different types of samples as shown in Figs. 14.4 and 14.6: We have successfully imaged selfassembled peptidic nanofibers, as well as fibrillar structures of cardiomyocytes (rat heart cells) using highly-uniform SERS substrates with 20$30 \mathrm{~nm}$ resolution (Fig. 14.4d). The results are promising, however, in its present form, a nanometer resolution Raman map can't be generated as this requires EMCCD cameras and a multi/hyperspectral imaging system. EMCCD cameras provide about 100-1,000 times greater sensitivity compared to the CMOS sensors 
(a)
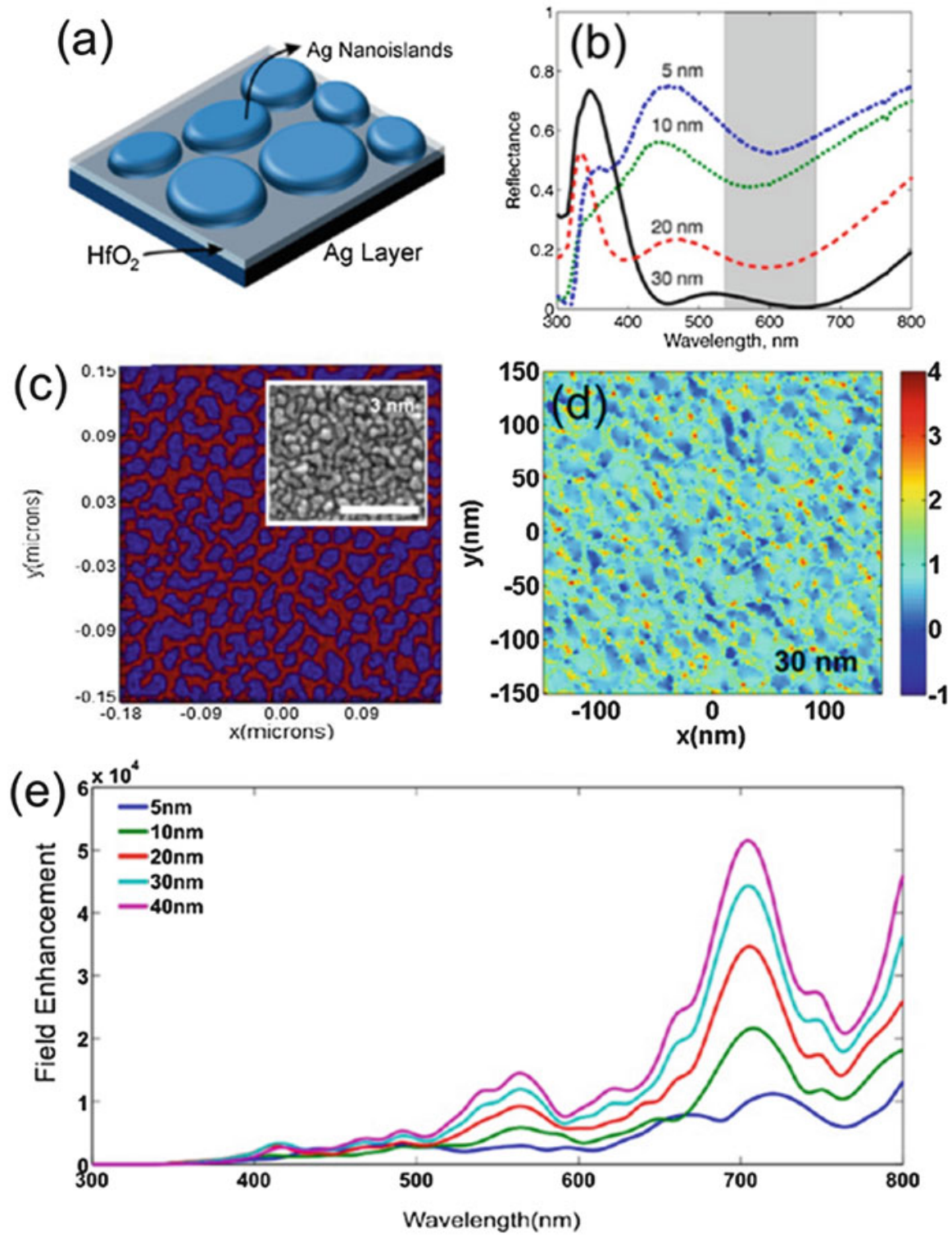

Fig. 14.5 Improved SERS substrates with single molecule sensitivity and high hot-spot density: (a) Ultrahin Ag films on dielectric layer on Ag substrate, segregates into nanoislands to form MIM surfaces. (b) These surfaces are omnidirectional superabsorbers if the dielectric thickness is around 30-40 nm. (c) A model surface is constructed for simulation using SEM micrographs (inset, scale bar $250 \mathrm{~nm}$ ). (d) Full field simulations show $|\mathrm{E}|^{2}$ field enhancement of $10^{4}\left(\log |\mathrm{E}|^{2}\right.$ is plotted). (e) Wavelength dependent field enhancement is shown for dielectric thicknesses of 5-40 nm. About $3 \times 10^{9}$ SERS enhancement is seen around $700 \mathrm{~nm}$ wavelength 

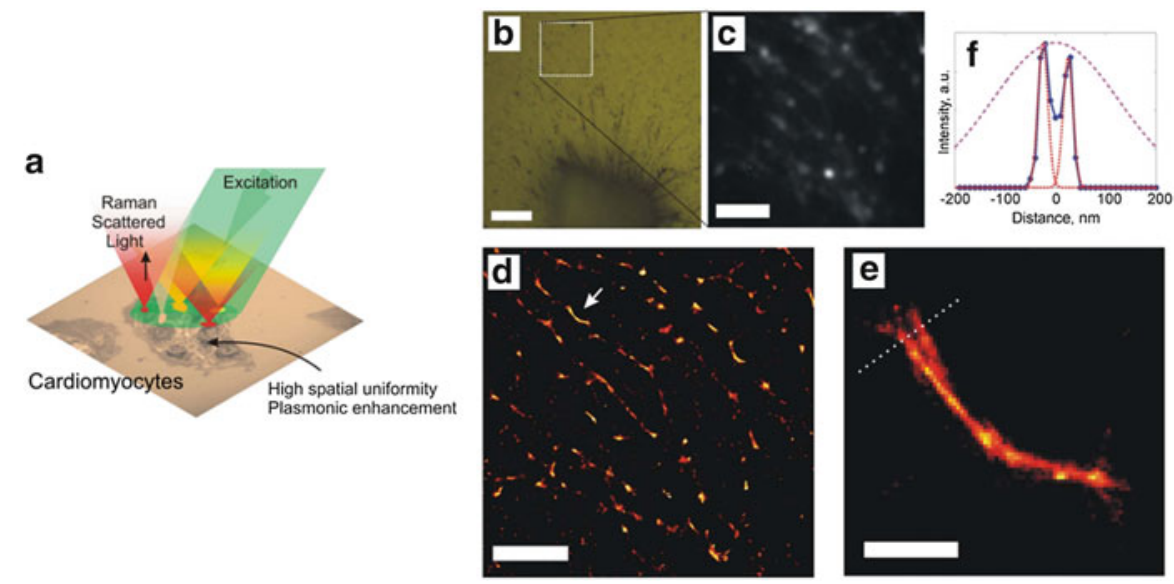

Fig. 14.6 Feasibility of SERS-STORM imaging. (a) Schematic description and (b) bright-field image of the edge of a cardiomyocyte on the plasmonic substrate. (c) A frame extracted from a video sequence of SERS images recorded using wide-field illumination and a low-cost CMOS camera, shows blinking SERS signal. (d) Stochastic reconstruction reveals fibrillar structure. (e) Detail of region shown by arrow in (d). (f) Line profile shows individual fibers with apparent diameter of $20 \mathrm{~nm}$, separated by $50 \mathrm{~nm}$. Diffraction limited profile is given by the dashed line. The reconstructed images use the whole integrated Raman spectrum, which can't currently be spectrally separated for chemically resolved HYPERSTORRM. The development effort aims to achieve such high resolution imaging, however with chemical specificity

used in acquisition of Figs. 14.4 and 14.6. EMCCDs have sufficient sensitivity which allow for the registration of signals from individual bands (which would contain 5-10\% of total optical power in a SERS blink). We demonstrate that the SERS signal can be acquired without optical damage to the chemical structure of biomolecular constructs (Fig. 14.6e). Therefore, although chemical resolution is not achieved in the preliminary results, they show that chemically specific HYPERSTORRM imaging is feasible.

The idea of hyperspectral imaging is tested as shown in Fig. 14.2 by using a one dimensional grating (as shown in Fig. 14.7). Clearly, one can separate the 0th order image from the 1st order image. In addition, when used with a SERS substrate as described earlier, blink events can be captured simultaneously in the 0th and 1 st order images. Spectral separation of the content of the blink events are visible. However, for final implementation, a two dimensional optimized grating must be used with a high sensitivity EMCCD camera. Also mathematical methods must be fully developed so as to achieve highest signal to noise ratio and spectral clarity.

Within a first order semi-classical approximation, the SERS photon generation areal density rate $\varphi\left(x, y, \omega_{s}, t\right)$ observed on a plasmonic substrate is a function of a large number of variables, such as spatial orientation of fields, molecular configurations, and hot spots. Also, the SERS signal depends on Raman excitation and scattering frequencies, $\omega_{\text {exc }}$ and $\omega_{s}$, as well as time $t$. The time dependence is observed to be strongly dependent on other parameters, such as excitation intensity, 

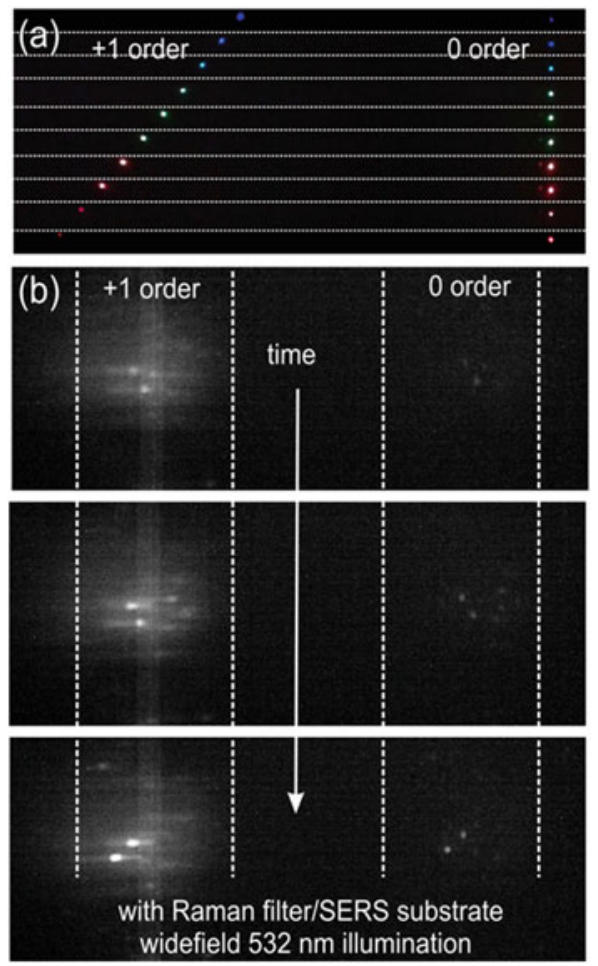

Fig. 14.7 Preliminary results on implementation of hyperspectral imaging of SERS signals. (a) Using a one dimensional transmission grating, 0th and 1st orders of a point source is imaged using the setup shown in Fig. 14.2a. The 0th order image does not shift with wavelength, whereas the 1st order image shifts due to diffraction. An RGB CMOS camera is used. (b) Consecutive frames are given from a video capture of SERS substrate treated with methylene blue, using a monochrome camera showing the 0th and 1st order regions. Again, the 0th order region exhibits discrete blinking spots, which can be used for localization. The 1st order region shows correlated spots, smeared in the horizontal direction due to spectral content of the SERS blinks. Initial results suggest that, using a high sensitivity EMCCD camera and an optimized 2D grating, more orders can be captured and be used for improved spectral/spatial resolution (Unpublished data)

local field enhancement factor, and chemical composition of imaged structures. At a given time $\mathrm{t}$, the infinitesimal intensity $d I=\varphi\left(x, y, \omega_{s}, t\right) d A$ generated at an area $d A$ at a location (x,y) from a molecular component $i$ is given as [5].

$d I_{i}\left(x, y, \omega_{s}, t\right)=Q I_{L}\left(x, y, \omega_{\text {exc }}\right) \varepsilon\left(\omega_{\text {exc }}\right)^{-1} \Omega \mathrm{S}_{\mathrm{i}}\left(\omega_{s}, t\right) N_{i}(x, y, t) E_{F}\left(x, y, \omega_{s}, \omega_{\text {exc }}\right) d A$

Where $\mathrm{S}_{\mathrm{i}}\left(\omega_{s}, t\right)=\frac{d \sigma_{i}\left(\omega_{s}, t\right)}{d \Omega}$ is the differential scattering cross-section of molecular component $i$, characterizing its Raman spectrum. Here, $Q$ is the overall optical collection and the detection efficiency; $I_{L}\left(x, y, \omega_{\text {exc }}\right)$ is the excitation power density; 
$\varepsilon\left(\omega_{\text {exc }}\right)$ is the photon energy at excitation frequency; $\Omega$ is the solid-angle collected by the objective; $N_{i}(x, y)$ is the areal density distribution of molecular component $I$; and $E_{F}\left(x, y, \omega_{s}, \omega_{e x c}\right)$ is the function describing the spatial distribution of SERS enhancement factor. Assuming spatially uniform illumination, $I_{L}\left(x, y, \omega_{\text {exc }}\right)=I_{L 0}$, a wide-field image can be formed using the SERS signal from a spatial pattern contained in the molecular layer $N_{i}(x, y)$ provided that the SERS enhancement distribution $E_{F}\left(x, y, \omega_{s}, \omega_{e x c}\right)$ is spatially constant or highly uniform. Although interference effects and speckle patterns are typically present during wide-field coherent illumination, uniform illumination assumption is reasonably satisfied for small dimensions. If the plasmonic modes responsible for Raman enhancement can be regarded as uncoupled or weakly coupled, hot spots can be thought of as operating independently. In such a case, regardless of the details of fluctuation mechanisms, the fluctuations originate from local effects which are uncorrelated. Such an optical signal, i.e. one with uncorrelated nanoscale spatial and temporal structure at the source, can be used for super-resolved stochastic imaging.

The simplest and most straight forward way of achieving multispectral STORM images is to reconstruct individual spectral channels separately and combine them by post processing. Mathematically, localization precision of point-like objects imaged in two-dimension is given by $\sigma_{x}^{2}=\frac{\mathrm{r}_{0}^{2}+\mathrm{q}^{2} / 12}{\mathrm{~N}}+\frac{8 \pi \mathrm{r}_{0}^{4} \mathrm{~b}^{2}}{\mathrm{q}^{2} \mathrm{~N}^{2}}$, where $\mathrm{r}_{0}$ is the standard deviation of the point spread function, $\mathrm{N}$ is the total number of photons collected, $\mathrm{q}$ is the size of an image pixel, and b is the background noise per pixel [20]. For single blink events resulting in $\sim 10^{5}$ SERS photons, the resolution is expected to be on the order of few nanometers. It is not clear if the filtering required for spectral separation will allow collection of $10^{5}$ SERS photons during a single blink event. Reduction of the collected photon number to $10^{3}$ SERS photons will limit the resolution to tens of nanometers. It may be required to use modified algorithms during extraction of spot locations based on data from multiple cameras. Techniques alternative to finding the distinct individual centroid locations exist for super-resolution imaging such as optical fluctuation imaging (SOFI) [21]. Inspired by such techniques, we propose to use a correlation based technique, not for centroid localization, for hyperspectral image separation.

One of the novelties in this work is the use of a two dimensional grating for hyperspectral imaging as shown in Fig. 14.2c. A two dimensional grating positioned in front of a camera will cause the image to split into 0th and higher order mode images. The higher order mode images will be spectrally separated. In the imaging configuration shown in Fig. 14.2, the 2D dispersion of the grating is characterized for orders $m$ and $n$ by a shift of the intensity of the spectral components of the 0th order image by $\alpha m \lambda$ and $\alpha n \lambda$ in $\mathrm{x}$ and $\mathrm{y}$ directions. The collection quantum efficiency and diffraction efficiency are assumed to be uniform. Also, the presence of the 2D grating is assumed not to cause distortions in higher order mode images, which is reasonable assumption for small diffraction angles. For the imaging configuration shown in Fig. 14.2d, the intensity distribution observed by the camera can be approximated as 
$I(x, y, t)=\sum_{m, n} \gamma_{m, n} \iiint_{x 0, y 0, \lambda} \delta(x-x 0-\alpha m \lambda) \delta(y-y 0-\alpha n \lambda) S(x 0, y 0, \lambda, t) d \lambda d x 0 d y 0$

Where $\gamma_{m, n}$ is the efficiency of the order, and $\mathrm{m}, \mathrm{n}, S(x 0, y 0, \lambda, t)$ is the source located at $(\mathrm{x} 0, \mathrm{y} 0)$ with spectral distribution given by a function of $\lambda$ and a time dependent intensity fluctuation. The diffraction limit can be incorporated by including a point spread function (PSF) to replace the delta functions $\delta(x) \delta(y) \stackrel{\Delta}{\rightarrow} \operatorname{PSF}(x, y) \triangleq$ $\exp \left(-\frac{x^{2}+y^{2}}{2 \sigma^{2}}\right)$. The intensity of the spectral function $S(x, y, \lambda, t)$ can be written as the product of a time independent spectral function $S(x, y, \lambda$,$) that depends on location$ (x,y), wavelength $\lambda$, and a time and location dependent random variable $T(x, y, t)$, as $S(x, y, \lambda, t)=S(x, y, \lambda) T(x, y, t)$. This can be justified by the experimental results shown in Fig. 14.7, where simultaneous measurement of spectral fluctuations and location dependent blinking events are recorded on a SERS substrate. In other words, we ignore small fluctuations of spectral positions of the Raman bands, and treat the blinking of the SERS signal as a temporal envelope that modulates the full SERS spectrum. Because the camera reads intensities that are absolute positive, it is more convenient to work with the time derivative of the intensity $\dot{S}(x, y, \lambda, t)=$ $S(x, y, \lambda) \dot{T}(x, y, t)$. We assume that $\langle\dot{T}(x, y, t)\rangle=0$, which says that the time average of the fluctuations is zero and $\left\langle\dot{T}(x, y, t) \dot{T}\left(x^{\prime}, y^{\prime}, t\right)\right\rangle=\delta\left(x-x^{\prime}, y-y^{\prime}\right)$, which explains that different locations are uncorrelated in time. We don't make any assumptions about the autocorrelation function of $\dot{T}(x, y, t)$, apart from the observation that the autocorrelation has a finite bandwidth and blink events occur with an average frequency on the order of a second.

We can write the intensity separated in orders $(m, n)$ as

$$
I^{m, n}(x, y, t)=\gamma_{m, n} \iiint_{x 0, y 0, \lambda} \delta(x-x 0-\alpha m \lambda) \delta(y-y 0-\alpha n \lambda) S(x 0, y 0, \lambda, t) d \lambda d x 0 d y 0
$$

Then the 0th order intensity is integrated over the spectrum, $I^{0,0}(x, y, t)=$ $\gamma_{0,0} \int_{\lambda} S(x, y, \lambda, t) d \lambda \triangleq \gamma_{0,0} S(x, y, t)$. The intensity for $(\mathrm{m}, \mathrm{n})=(1,0)$ will be $I^{1,0}(x, y, t)=\gamma_{1,0} \int_{\lambda} S(x-\alpha \lambda, y, \lambda, t) d \lambda$. The problem is to determine $S(x, y, \lambda)$.

We assume that the fluctuations from different molecules are uncorrelated, i.e. use $\left\langle\dot{T}(x, y, t) \dot{T}\left(x^{\prime}, y^{\prime}, t\right)\right\rangle=\delta\left(x-x^{\prime}, y-y^{\prime}\right)$, and get

$$
\int_{t} \dot{S}(x, y, \lambda, t) \dot{S}\left(x^{\prime}, y^{\prime}, \lambda, t\right) d t=\delta\left(x-x^{\prime}, y-y^{\prime}\right) S(x, y, \lambda)
$$


Where $S(x, y, \lambda)$ is the time average spectrum of molecule at position (x,y). Using a similar approach, we can calculate the correlation of pixel in the 0 th and 1st order image regions, to find $\int_{t} \dot{I}^{0,0}(x, y, t) \dot{I}^{1,0}\left(x^{\prime}, y^{\prime}, t\right) d t=\delta\left(x-x^{\prime}+\alpha \lambda\right) \delta\left(y-y^{\prime}\right)$ $S(x, y, \lambda) \int_{\lambda} S\left(x^{\prime}, y^{\prime}, \lambda\right) d \lambda$. Therefore, the correlations of pixels $(\mathrm{x}, \mathrm{y})$ in the 0 th order image with the $\left(x^{\prime}, y^{\prime}\right)=(x-\alpha \lambda, y)$ in the $(1,0)$ order image result in intensities proportional to $S(x, y, \lambda)$ the $\lambda$ spectral component of the corresponding source pixel at $(\mathrm{x}, \mathrm{y})$. A similar approach can be used to improve convergence of the correlations using other orders. A more detailed formalism must be developed if the Raman band frequencies are assumed to fluctuate as well as the intensities.

\subsection{Conclusions}

In summary, we discuss a novel form of label-free super-resolution imaging, namely hyperspectral stochastic optical reconstruction Raman microscopy, which has the potential to provide chemically resolved Raman maps with nanometric resolution. The technique relies on SERS, and has inherent limitations: The resolution is limited by hot-spot spacing of the plasmonic surfaces, and observations are limited to the surface plane of the plasmonic substrates. Still, the method can be used to study biologically relevant specimens, such as membrane phenomena.

Acknowledgments The author acknowledges TUBITAK Grant 111M344 and EU-FP7 NanobacterphageSERS for financial support.

\section{References}

1. Wang MC, Min W, Freudiger CW, Ruvkun G, Xie XS (2011) RNAi screening for fat regulatory genes with SRS microscopy. Nat Methods 8:135-138

2. Steidtner J et al (2008) Tip-enhanced Raman spectroscopy and microscopy on single dye molecules with $15 \mathrm{~nm}$ resolution. Phys Rev Lett 100:236101

3. Jeanmaire DL, Van Duyne RP (1977) Surface Raman spectroelectrochemistry: part I. Heterocyclic, aromatic, and aliphatic amines adsorbed on the anodized silver electrode. J Electroanal Chem Interfacial Electrochem 84:1-20

4. Albrecht MG, Creighton JA (1977) Anomalously intense Raman spectra of pyridine at a silver electrode. J Am Chem Soc 99:5215-5217

5. Stiles PL, Dieringer JA, Shah NC, Van Duyne RR (2008) Surface-enhanced Raman spectroscopy. Annu Rev Anal Chem 1:601-626

6. Nie S, Emory SR (1997) Probing single molecules and single nanoparticles by surfaceenhanced Raman scattering. Science 275:1102-1106

7. Kneipp K et al (1997) Single molecule detection using surface-enhanced Raman scattering (SERS). Phys Rev Lett 78:1667-1670 
8. Kneipp K, Wang Y, Dasari RR, Feld MS (1995) Approach to single molecule detection using surface-enhanced resonance Raman scattering (SERRS): a study using Rhodamine 6G on colloidal silver. Appl Spectrosc 49:780-784

9. Kneipp K et al (1998) Surface-enhanced Raman scattering (SERS) - a new tool for single molecule detection and identification. Bioimaging 6:104-110

10. Le Ru EC, Etchegoin PG (2012) Single-molecule surface-enhanced Raman spectroscopy. Annu Rev Phys Chem 63:65-87

11. Rust MJ, Bates M, Zhuang X (2006) Sub-diffraction-limit imaging by stochastic optical reconstruction microscopy (STORM). Nat Methods 3:793-796

12. Stranahan SM, Willets KA (2010) Super-resolution optical imaging of single-molecule SERS hot spots. Nano Lett 10:3777-3784

13. Cang $\mathrm{H}$ et al (2011) Probing the electromagnetic field of a 15-nanometre hotspot by single molecule imaging. Nature 469:385-388

14. Balzarotti F, Stefani FD (2012) Plasmonics meets far-field optical nanoscopy. ACS Nano 6:4580-4584

15. Perney NMB et al (2006) Tuning localized plasmons in nanostructured substrates for surfaceenhanced Raman scattering. Opt Express 14:847-857

16. Ayas $\mathrm{S}$ et al (2012) Raman enhancement on a broadband meta-surface. ACS Nano 6:6852-6861

17. Ayas S, Cinar G, Dana A et al (2013) Label-free nanometer-resolution imaging of biological architectures through surface enhanced Raman scattering. Sci Rep 3:2624

18. Ayas $S$ et al (2014) Counting molecules with a mobile phone camera using plasmonic enhancement. ACS Photonics 1:11

19. Fisher J, Baumback MM, Bowles JH, Grossmann JM, Antoniades JA (1998) Comparison of low-cost hyperspectral sensors. In: Proceedings of SPIE 3438, Imaging Spectrometry IV, 23. doi:10.1117/12.328112

20. Hess ST, Girirajan TPK, Mason MD (2006) Ultra-high resolution imaging by fluorescence photoactivation localization microscopy. Biophys J 91:4258

21. Dertingera T, Colyera R, Iyera G, Weiss S, Enderlein J (2009) Fast, background-free, 3D superresolution optical fluctuation imaging (SOFI) PNAS 10652 22287-22292 